\title{
Clock Synchronization Methods of Electric Meters Based on Wireless Communication
}

\author{
Ying SUN ${ }^{\mathrm{a}, 1}$,Zhipeng SU ${ }^{\mathrm{b}}$, Qiong WU ${ }^{\mathrm{a}}$, Feiou YUa, Ying ZHAO ${ }^{\mathrm{a}}$, and Enzhen $\mathrm{HOU}^{\mathrm{c}}$ \\ ${ }^{a}$ Marketing Department, Guangzhou Power Supply Bureau of Guangdong Grid Co, \\ Guangzhou, China \\ ${ }^{\mathrm{b}}$ Guangzhou Power Supply Bureau of Guangdong Grid Co, Guangzhou, China \\ ${ }^{\mathrm{c}}$ Network Information Company, China Energy Engineering Group Guangdong \\ Electric Power Design Institute Co., Ltd, Guangzhou, China
}

\begin{abstract}
Aiming at the inaccurate clock calibration of electric meters and the shortcomings of traditional clock synchronization methods, this article briefly summarizes the reasons of inaccurate meter clocks, and proposes a method of electric meter clock calibration based on wireless communication, which solves the problem of meter clock synchronization accuracy. It is verified by field test. The test results show that the error of electric meters clock after clock calibration can be controlled at the second level. And the data error of collected import active electrical energy is smaller, which meets the requirements of real-time analysis of electricity spot trading and other businesses, providing more accurate data to ensure the results reliability.
\end{abstract}

Keywords. Electric meters, Wireless communication, Clock synchronization, Electricity spot trading

\section{Introduction}

The development of power system has higher requirements for the clock accuracy of electric meters. The accuracy of clock is closely related to the accuracy of electric meters. The clock disorder of meters will cause a series of problems such as the failure implementation of electricity price adjustment task, the untimely switching of peak and valley periods, and the failure of remote reading of the user's electrical energy data. The implementation of national electricity price policy on the company, the statistics of user's electricity consumption information, the calculation of users electricity bills based on stepped electricity price, accurate calculation of line losses during the same period, and data analysis of various majors in the power grid will be seriously affected. Therefore, accurate and efficient clock calibration methods are of great significance to support the deepening application of power data.

Statistics show that as of 2018, the clock deviation of more than 1 min accounted for $19 \%$ of electricity meters in operation in a province. And the clock deviation of more than 5 minutes accounted for 13\% [1]. According to statistics of the Guangdong Electric Power Trading Center, the clock deviation of more than 5 minutes accounted for $94.8 \%$ [2]. However, the load measurement cycle in electricity spot market is usually 15 minutes

${ }^{1}$ Corresponding Author, Ying SUN, Marketing Department, Guangzhou Power Supply Bureau of Guangdong Grid Co, Guangzhou, China; E-mail: 410050938@qq.com. 
or less[3]. The clock deviation of 5 minutes is equivalent to at least $33 \%$ deviation in each statistical period, which will greatly affect the settlement results of electricity spot trading. According to the above statistical data, most electric meters can only maintain minute-level clock accuracy. To achieve the second-level accuracy of clock synchronization for electric meters, a new clock calibration method is required.

The traditional clock synchronization strategy generally adopts the method of batch test of all electric meter clocks. For clock deviations between 1 and 5 minutes, it use broadcast clock calibration. And use point-to-point clock calibration for clock drift more than 5 minutes[1]. This clock synchronization method is given a large operating load to the master station of acquisition system. It seriously occupies the acquisition system resources, and completes a long execution cycle of the clock calibration. And the timeliness is poor.

In order to improve the accuracy of electric meter clock, this article firstly analyses the current status of electric meter clock errors and the high requirements of new business on the accuracy of electric energy meter clock. Based on the current situation, a wireless communication electric meter clock calibration method is proposed. The clock calibration method proposed in this paper uses wireless communication to realize the transmission of time messages and the error processing method for the time message of electric meters, which can improve the clock synchronization accuracy of electric meters without changing the electric meters.

\section{Clock Synchronization Methods for Electric Meters}

The accuracy of electric meter clocks is affected by many factors: clock circuit scheme (whether an independent clock chip is used), component selection and quality, clock chemistry, and PCB design and production [4]. Many of the above factors, combined with different environmental factors, cause the drift of the meter clocks. To solve the problem from meters source, it is necessary to modify the design of meters, which directly increases the meters cost. For example, the use of a clock component with a built-in temperature-compensated crystal oscillator can reduce clock errors. Ideally, the clock error can be reduced to within 0.3 seconds per day. Even if a high-precision clock chip is used, the clock error still accumulates over time. And a few minutes of clock error will occur after one year. Therefore, even if the clock adopts high-quality clock circuits and components, it still uses radio waves to synchronize the clock to ensure clock accuracy. In addition, electric meters using high-cost clock solutions cannot solve the clock problems of massive existing equipment.

Using high-frequency and high-precision clock calibration methods can better solve the problem of meters clock drift, improving clock calibration accuracy, and avoiding clock errors caused by communication delays, multi-level clock synchronization and equipment failures. Choosing a high-precision clock source can increase the calibrating frequency and reduce the requirement for the meter clock circuit to maintain clock accuracy for a long time. Different working environments of electric meters have different requirements on the frequency of clock calibration. A typical clock crystal has an accuracy of ppm at room temperature, or an error of seconds per day. In order to increase the frequency of clock calibration, it can be adjusted once per hour.

For clock battery failure, the main reason that affects the clock error of meters [1], clock calibration can also improve availability of the failed meters. The clock battery of 
electric meters is only used as a backup power source for the clock circuit when there is no mains power [5]. The failure of clock battery causes the time to restore the factory configuration after power-on of the meter every time. The clock calibration can ensure the correct time of electric meters in the working state.

In the current metering automation system, the collection terminal is equipped with a $4 \mathrm{G}$ wireless communication module, which can obtain the absolute time from communication base station. In this paper, according to the business characteristics, the collection terminal actively uses the wireless communication network base station to synchronize the clock to reduce the load of collection master station [6]. In this way, the collection terminal can rely on the communication module to become a secondary clock source, which improves the accuracy of clock synchronization on the one hand, and also improves the frequency of time service and clock synchronization on the other hand. It can be realized by upgrading the collection terminal software. Compared with GPS and other clock calibration methods, it is not necessary to add or modify existing equipment. On the other hand, to obtain the base station time from $4 \mathrm{G}$ wireless communication module, there is no need to consider the signal reception problems of other clock systems. For example, GPS needs to lead the antenna to the window or outdoors.

\subsection{Absolute Clock Synchronization}

The architecture of absolute clock synchronization is illustrated as in figure 1. Electric meters connect to clock synchronization device through RS485 or Ethernet interface (if the electricity meter has an Ethernet interface), and require absolute clock time. The clock synchronization device could integrate or connect to the absolute clock source, such as GPS or Beidou[4]. Under this structure, the clock source device is GPS or Beidou to ground. And the clock source is the meter's clock synchronization device.

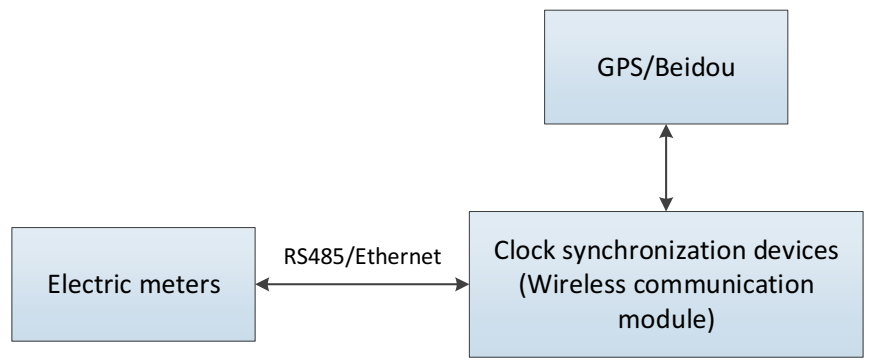

Figure 1. Wireless communication based clock synchronization integrating GPS or Beidou.

The clock synchronization device of electric meters includes:

- The internal clock synchronization module is used to obtain the absolute time and synchronize the internal clock according to the absolute time.

- The message receiving module sends a time adjustment message to the electric meter at the first time through a pre-set communication protocol. And it receives the second adjustment message fed back by the electric meters at the second time. The first and second time is the internal clock time.

- Obtain the processing delay of the first clock synchronization messages according to the first and second time and the transmission delay. According to 
the processing delay, transmission delay, pre-determined message assembly time, and the time of internal clock get the calibration time;

- Send a time calibration message to the electric meter, where the time calibration message contains the calibration time to synchronize the local clock of the electric meter.

\subsection{Wireless Communication Based Clock Synchronization}

The meter clock device does not have a GPS or Beidou absolute clock source, but it has a wireless communication module. The architecture of relative clock synchronization is illustrated as in figure 2. The meters connect to clock synchronization devices through RS485 or Ethernet interface (if the meter has an Ethernet interface). The meter clock synchronization devices establishes wireless communication between the wireless communication module and base station, and obtains the absolute time from the base station. That is, the clock source of electricity meters to the ground device is the wireless communication base station. And the clock source of electricity meters is the electric meter clock synchronization devices.

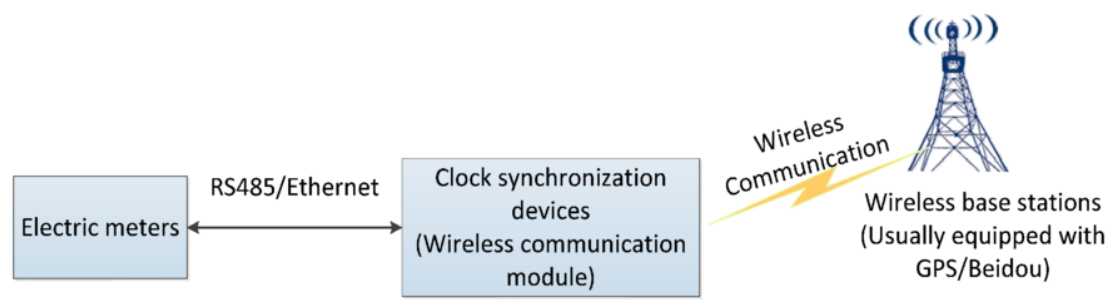

Figure 2. Wireless communication based clock synchronization from wireless base stations.

After obtaining the high-precision clock source, the clock synchronization of the electric meter is realized by steps. The execution process of the electric meter clock synchronization is shown in figure 3 and figure 4. Except for the method of acquiring absolute time, the other processes are the same. There don't describe it again separately. The following clock synchronization process is executed regularly. And it can be configured as a minute, hour, or daily execution cycle. The function of meter clock device is built into the wireless communication module to complete. 


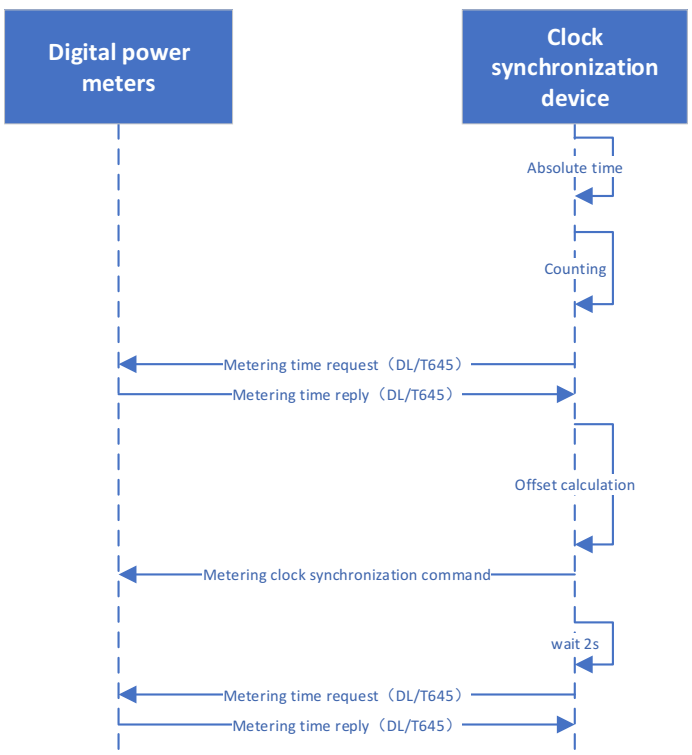

Figure 3. clock synchronization with clock source.

The wireless communication meters clock synchronization process:

- The meter clock synchronization devices (communication terminals) send a request to the base station and obtain the absolute time $T_{0}$ of the wireless communication base station.

- Records the first time $T_{1}$ which send the absolute time packet by the communication terminal to meters. The second time for the terminal to receive the meter clock messages is $T_{2}$.

- Assuming that the transmission delay of the communication terminal to the meter is $\delta_{1}$. And the transmission delay of the message from the meter to the terminal is $\delta_{2}$.

- After parsing the message, the meter time is $T_{3}$.

Next, calculate the time deviation. The time difference of a packet round trip between from the communication terminal and the meter is $\mu_{1}=T_{2}-T_{1}$;

Delay in processing the first packet: $\mu_{2}=T_{2}-T_{1}-\delta_{1}-\delta_{2}$

the actual meter time: $T_{4}=T_{3}+\delta_{2}$

clock calibration packet is the error of the actual meter time and the absolute time: $\mu_{2}=T_{4}-T_{0}$ 


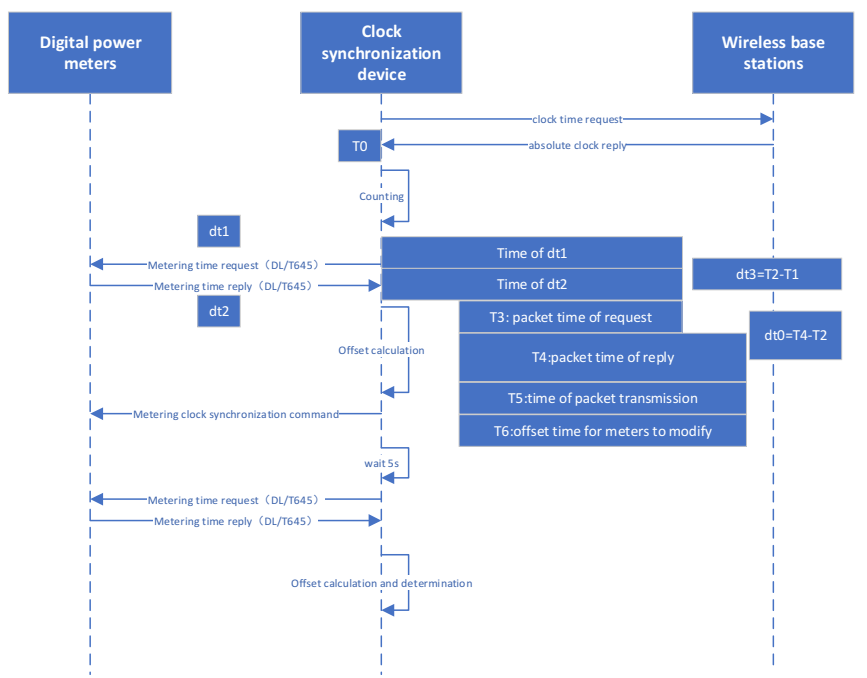

Figure 4. clock synchronization based on wireless communication.

The establishment of a safe and reliable clock synchronization network is the foundation of modern power systems. By using Beidou/GPS as the clock source combined with modern wireless communication systems, a communication clock synchronization network that is conceptually independent from the business network is established to meet the requirements for precise frequency and clock synchronization in the communication, measurement and control systems of the power industry. The following figure shows the detailed steps of using the wireless communication system to calibrate the meter, and the meaning of each time data.

\section{Experiments and Results}

The establishment of a safe and reliable clock synchronization network is the foundation of modern power systems. By using Beidou/GPS as the clock source combined with modern wireless communication systems, a communication clock synchronization network that is conceptually independent from the business network is established to meet the requirements for precise frequency and clock synchronization in the communication, measurement and control systems of the power industry. The following figure shows the detailed steps of using the wireless communication system to calibrate the meter, and the meaning of each time data.

The project team carried out the related work of electricity spot trading meter data collection in Guangzhou. The data items collected by electric meter and the collection frequency are flexibly configured and sent to the collection device by the master station. The collection device actively collects the meter data and uploads it to the master station data receiving program according to the collection rules of the configuration file. The master station side decodes the meter protocol and stores the data in the database.

This article selects a customer from a shopping mall of China Southern Power Grid Corporation Limited for field testing from 8:40 am to 14:30 pm on business days. This test uses two SIM cards, one of which can be connected to the test master station. But the base station time cannot be obtained. The other one can obtain the base station time, 
but cannot connect to the test master station. When a normal SIM card is used to register the wireless module with the base station. The base station time will be obtained. And then the time will be adjusted with the base station at regular intervals. The communication terminal can obtain the time from the base station. And the communication terminal can calculate the difference between the time of the electric meter and the time of the communication terminal in real time.

According to the actual test of the total import active electrical energy, the communication terminal time and the meter time, the clock drift degree between before and after the clock calibration was recorded and compared. And the time drift diagrams are shown in figure 5 and figure 6 below.

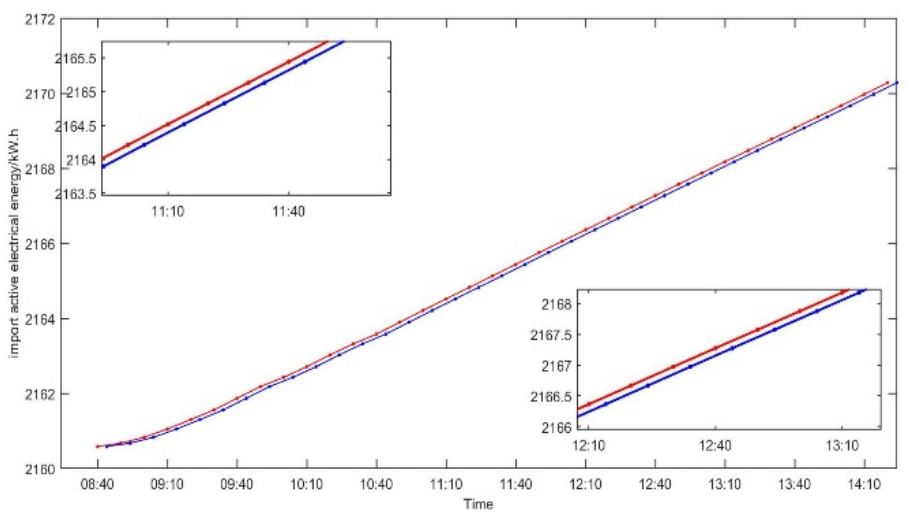

Figure 5. Clock drift diagram before clock calibration。

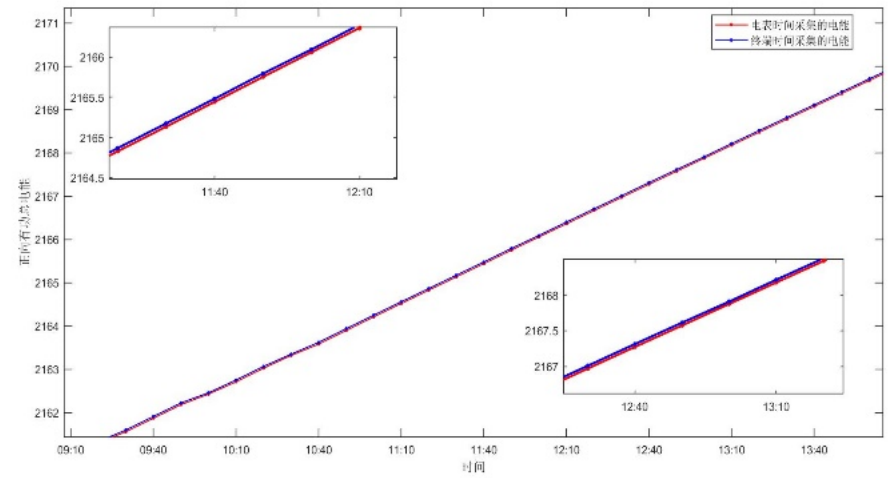

Figure 6. Clock drift diagram after clock calibration.

Through the comparative analysis of figures 5 and 6 , it can be seen that the drift between the meter time and the time of the communication terminal before clock synchronization is significantly greater than the drift after calibration, which is corrected by the wireless communication base station. The meter time is closer to the absolute time obtained by the terminal. And the time error can reach the second level after calibration according to the obtained data, which meets the real-time requirements of the electricity spot transaction business. It can be seen from the above figure that the time of electric meters and the time of communication terminals are not completely consistent. And there 
is a small deviation between them. The inaccuracy of the collected import active electrical energy data is caused by the clock drift. figures 7 and 8 below show the error of the total import active electrical energy before and after the clock synchronization.

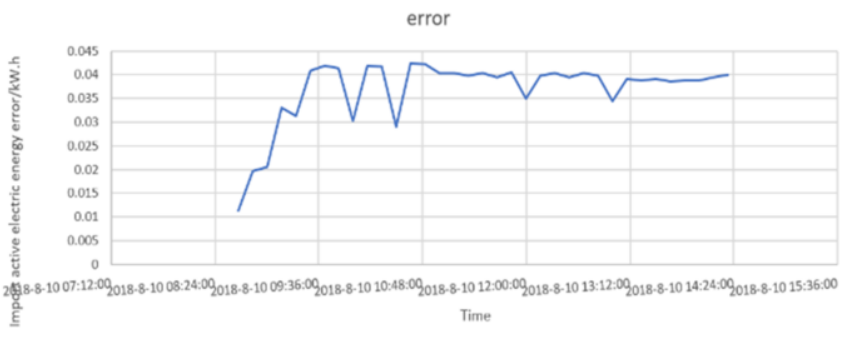

Figure 7. Import active electrical energy error before clock synchronization.

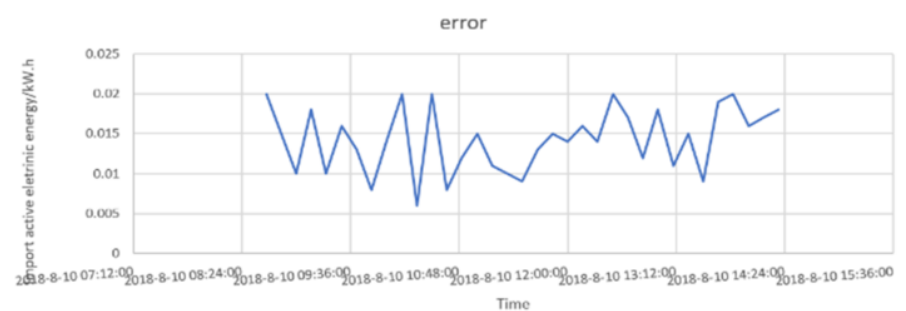

Figure 8. Import active electrical energy error after clock synchronization.

By comparing figure 7 and figure 8 , we can see that the error of the total import active electrical energy before clock calibration fluctuates around 0.04. But the error of the total import active electrical energy fluctuates around 0.015 after clock calibration.

As a result, the data obtained by clock calibration is more accurate, which provides a more accurate data basis for subsequent electricity spot trading business and electricity bill settlement analysis. And the result will be more reliable.

\section{Conclusion}

This paper proposed two wireless communication clock synchronization methods to maintain the clock synchronization accuracy of electric meters at levels of seconds, thus guaranteeing the successful real-time metering data collections. The method proposed in this paper does not need to upgrade the existing collection terminals. And it uses external equipment to improve the accuracy of meter clocks. It provides support for businesses that require high meter clock accuracy such as electricity spot trading. Accurate clock calibration can effectively improve the system's clock synchronization efficiency, reducing the burden of master station servers and saving cost of electric meters due to clock failure. As the clock synchronization equipment is restricted by the DLT645 protocol, the clock calibration can only be used once a day. In the future, we can consider coordinating meter manufacturers to open more free time calibration authority on the second RS485 port, which can achieve high-frequency time calibration. If the cellular base station time synchronization method is approved, the existing collection terminal can be upgraded to support the base station timing, and the collection terminal can use a 
high-precision time source to calibrate the electric meter. This method only needs to upgrade the software of the existing collection terminal, without modifying or adding new equipment.

\section{Acknowledgements}

This study was supported by China Southern Power Grid Key Technology Project (080006KK52200001).

\section{References}

[1] WANG Hongxi, etc. 2014 Research and Application of Precise Timing Method for Smart Meter[J], Hebei Electric Power, vol. 39(2), pp. 22-25.

[2] Gong Xueliang, etc. 2019 Influence of Meter Clock Drift on Electricity Spot Market Settlement[J], Southern Power System Technology, vol. 13(10), pp. 87-94.

[3] Qian Lijun, etc. 2014 Problem Analyses and Countermeasures of the Real Time Clock in Smart Meters[J], Electrical Measurement \& Instrumentation, vol. 51(13), pp. 29-32.

[4] Liu Yin, etc. 2016 Analysis of meter clock problem caused by abnormal automatic time synchronization of acquisition terminal[J], Electronic Technology \& Software Engineering, vol. 13, p. 131.

[5] Liu Chao, and Du Yue. 2018 Season Analysis and Treatment Related to Time Clock Abnormality in Intelligent kWh Meters[J], Rural Electrification, vol. 12, pp. 78-79.

[6] OuYang Mengni, etc. 2019 Research on Marketing Big Data Accuracy in Electric Energy Meter Clock Management[J], Digital Technology \& Application, vol. 37(7), pp. 70-71. 